\title{
Publisher Correction: Chlorophyll $f$ synthesis by a super-rogue photosystem II complex
}

Joko P. Trinugroho (D), Martina Bečková, Shengxi Shao (D), Jianfeng Yu, Ziyu Zhao, James W. Murray, Roman Sobotka, Josef Komenda (1) and Peter J. Nixon (1)

Correction to: Nature Plants https://doi.org/10.1038/s41477-020-0616-4, published online 9 March 2020.

In this Letter originally published, in Fig. 1b, the arrow associated with the label 'PsbF' was missing and there was an erroneous arrow in the top left corner. The original and corrected figure is shown below. In the bottom graph of Fig. 1d, the values $0,1,2$ and 3 were missing from the $y$ axis, as was the $x$ axis label 'Time (min)' and the label 'Chl $a$ ' for the peak of the curve. Furthermore, in Fig. 2c, the label ' $\Delta \mathrm{D} 1$ ' should have read 'His-CP47/ $\Delta \mathrm{D} 1$ '. These errors have now been corrected.
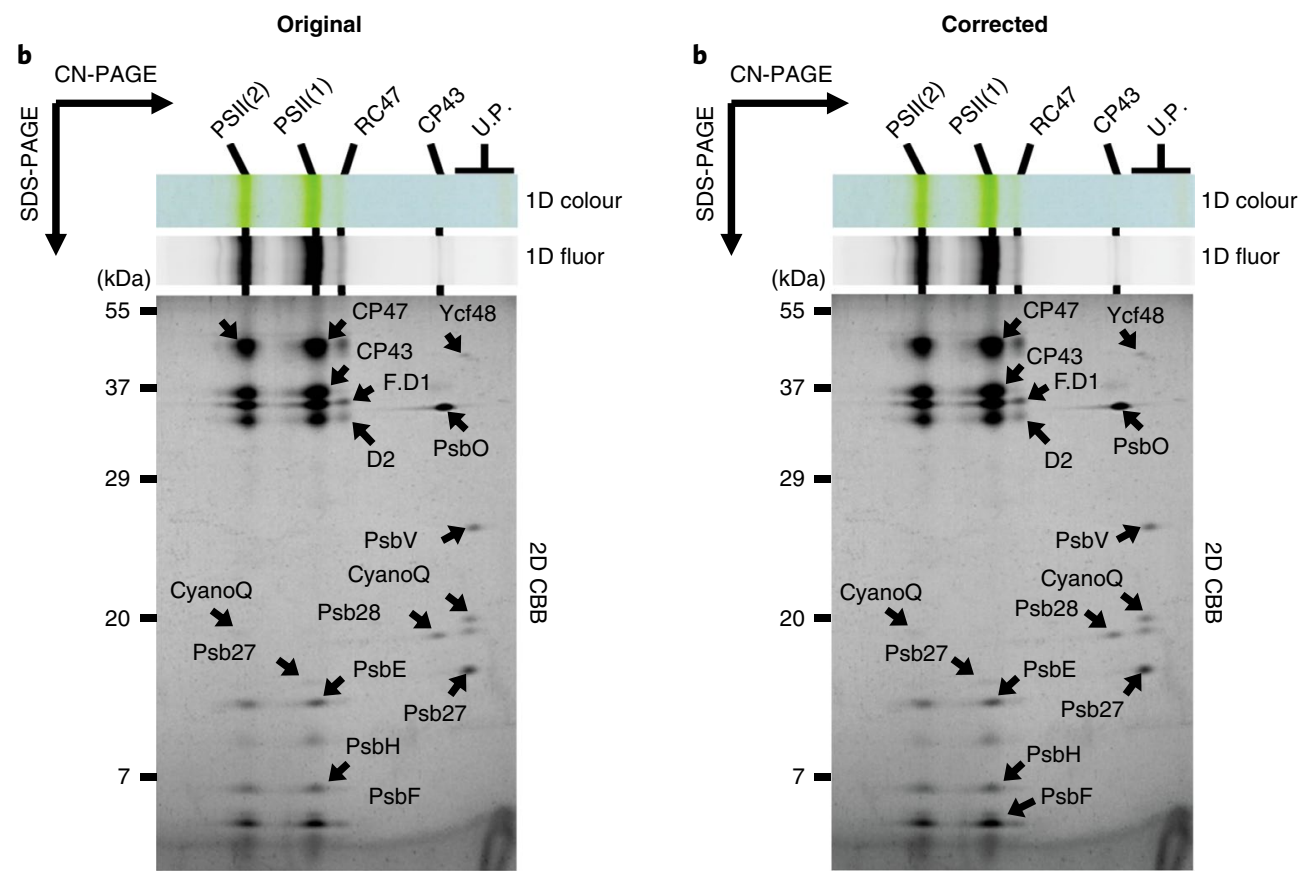

Fig. 1b | Original and corrected.

Published online: 3 April 2020

https://doi.org/10.1038/s41477-020-0649-8

(c) The Author(s), under exclusive licence to Springer Nature Limited 2020 